\title{
Thermodynamically consistent equation of state for an accreted neutron star crust
}

\author{
M.E. Gusakov and A.I. Chugunov \\ Ioffe Institute, St.-Petersburg, Russia
}

(Dated: April 10, 2020)

\begin{abstract}
We study equation of state (EOS) of an accreting neutron star crust. Usually, such EOS is obtained assuming (implicitly) that the free (unbound) neutrons and nuclei in the inner crust move together. We argue, that this assumption violates the condition $\mu_{n}^{\infty}=$ const, required for hydrostatic (and diffusion) equilibrium of unbound neutrons ( $\mu_{n}^{\infty}$ is the redshifted neutron chemical potential). We construct a new EOS respecting this condition, working in the compressible liquid-drop approximation. We demonstrate that it is close to the catalyzed EOS in most part of the inner crust, being very different from EOSs of accreted crust discussed in the literature. In particular, the pressure at the outer-inner crust interface does not coincide with the neutron drip pressure, usually calculated in the literature, and is determined by hydrostatic (and diffusion) equilibrium conditions within the star. We also find an instability at the bottom of fully accreted crust that transforms nuclei into homogeneous nuclear matter. It guarantees that the structure of fully accreted crust remains self-similar during accretion.
\end{abstract}

Introduction.- Neutron stars (NSs) are the densest objects in the Universe. The composition of their deepest layers (inner core) is uncertain and is considered as the main mystery of the NS physics [1]. In contrast, it is believed that the composition of their outer layers, the so called NS crust, is relatively well-known. The outer region of the neutron star crust, referred to as the outer crust, is composed of atomic nuclei on the neutralizing background of degenerate, almost ideal electron gas. In the deeper layers, called the inner crust, the unbound neutrons are also present $[1,2]$. With the subsequent increase of the density, the crust ends and we reach the outer core, which (in the vicinity of the crust-core boundary) is composed of neutrons $(n)$, protons $(p)$, and electrons $(e)$.

The only way to check whether theoretical models of superdense matter are reliable is to confront them with NS observations. One of the most promising possibilities in this regard is to study accreting NSs, which are observed in binary systems with the active mass transfer from a companion star. For some of these sources the accretion process is transient, and in quiescent periods $\mathrm{X}$-ray telescopes are able to detect the thermal emission from the NS surface, revealing that it is heated up by accretion [3-6]. It is generally believed, that the heating is caused by the non-equilibrium nuclear reactions, which are initiated in the crust as it is compressed under the weight of newly accreted material. Obviously, adequate interpretation of observations requires a reliable model describing this process (see [7] for a recent review). A similar process can also be important for reheating of millisecond pulsars [8].

Starting with Ref. [9], a number of authors study the evolution of an accreted element as it compresses and sinks deeper and deeper towards the NS core in the course of accretion. Some of them used a one-component approximation [10-14], while others used reaction networks, allowing for mixtures of different nuclei [15-18]. They ap- plied either liquid-drop models [10-13, 15, 18], or up-todate theoretical atomic mass tables $[16,17]$, or detailed extended Thomas-Fermi calculations, allowing for the existence of unbound neutrons [14]. The main common feature of all these works is that they follow compositional changes associated with reactions induced by the increasing pressure inside an accreted fluid element ('traditional approach'). Such consideration would be clearly applicable if we compress uniform infinite matter. But in reality the inner crust is not uniform, so that unbound neutrons can travel between different layers to lower the system energy [19].

The traditional approach was known to lead to jumps of the neutron chemical potential $\mu_{n}$ at the phase transitions, which are especially pronounced in the onecomponent approximation and considerably soften if mixtures of nuclei are allowed for [15]. However, these effects were typically considered as a local inconsistency, which, likely, does not affect the global properties of the accreted crust. In this Letter we show that it is not the case and that allowing unbound neutrons to move independently of nuclei has a dramatic effect on the crust composition and equation of state (EOS). We construct the corresponding EOS within the compressible liquid drop model (CLDM), which ignores pairing and shell effects. This EOS is fully thermodynamically consistent, in particular, $\mu_{n}$ in the inner crust is continuous and, moreover, satisfies the hydrostatic (and diffusion) equilibrium condition, $\mu_{n}^{\infty}=$ const (see below), where $\mu_{n}^{\infty} \equiv \mu_{n} \exp (\nu / 2)$ is the redshifted $\mu_{n}$, and $\nu=2 \phi / c^{2}$ ( $\phi$ is the gravitational potential and $c$ is speed of light $[1])$.

The calculated EOS significantly differs from EOSs obtained within the traditional approach, being very close to the EOS of catalyzed crust.

In Ref. [20] we demonstrate additional inconsistency of the traditional approach: It leads to strong violation of the force balance equation for nuclei (gravitational and 
electric forces are both directed downwards) in a few rather extended regions of the inner crust, thus revealing inconsistency of the traditional approach from another point of view.

Equilibrium condition for unbound neutrons. - Neutrons, not bound to nuclei, exist in the inner NS crust. Except for a narrow layer of width $L$ near the outerinner crust interface $\left(L \lesssim 5 \mathrm{~m}\right.$ for $\left.T=5 \times 10^{8} \mathrm{~K}\right)$, they are superfluid (e.g., Ref. [2]), and move with the velocity $V_{s n}$, governed by the (linearized) superfluid equation, $m_{n} \partial V_{s n} / \partial t=-\nabla \mu_{n}^{\infty}$, where $m_{n}$ is the neutron mass (see, e.g., Refs. [21-23]). The hydrostatic equilibrium implies $\mu_{n}^{\infty}=$ const as a necessary condition in the whole region of neutron superfluidity.

In the narrow nonsuperfluid layer the typical diffusion time, $\tau^{D} \sim L^{2} / D \lesssim 3 \times 10^{6} \mathrm{~s}$ (the neutron diffusion coefficient $D$ is estimated in analogy to Ref. [24]), is much smaller than the replacement timescale of this layer by accretion, $\sim \rho L / \dot{M} \gtrsim 2 \times 10^{9}$ s (we take $\rho \sim 4.3 \times 10^{11} \mathrm{~g} \mathrm{~cm}^{-3}$ for the density and assume that $\dot{M}$ equals the local Eddington accretion rate, $\dot{M} \sim 10^{5} \mathrm{~g} \mathrm{~cm}^{-2} \mathrm{~s}^{-1}$ ). As a result, unbound neutrons in the non-superfluid layer should be, to a good approximation, in diffusion equilibrium: $\mu_{n}^{\infty}=$ const.

Three crustal EOSs .- Typical temperature in the crust of accreting NSs is $T \lesssim 5 \times 10^{8} \mathrm{~K}$ and have a minor effect on EOS [1], so below we shall work in the approximation of $T=0$. As discussed above, neutron Hydrostatic and Diffusion (nHD) equilibrium conditions imply $\mu_{n}^{\infty}=$ const in the whole inner crust (we assume that the region of neutron superfluidity extends to the crust-core boundary). To illustrate importance of this condition let us consider three EOSs: catalyzed (ground state) EOS, which is believed to describe pristine NS crust and two EOSs for accreted NS crust: (i) traditional, which completely disregards neutron diffusion (denoted as 'Trad' EOS) and (ii) new EOS that respects the nHD condition (denoted as 'nHD' EOS). For simplicity we apply CLDM, in which nuclei are described as liquid drops, located at the center of the spherical Wigner-Seitz (WS) cells $[1,2,25]$. We ignore a possible layer of nonspherical nuclei in the vicinity of the crust-core boundary (for EOSs based on SLy4 energy density functional, employed here, this layer is absent $[26,27])$. We also assume that the proton drip does not occur in the crust, which is true for all numerical models discussed here. The model is parametrized by the number densities $n_{n i}, n_{p i}$, and $n_{n o}$ for, respectively, neutrons and protons inside, and neutrons outside nuclei; the neutron skin surface density $\nu_{s}$; and the volume $V_{c}$ of WS cell, as well as by the (proton) radius $r_{p}$ of a nucleus. In addition, it is useful to introduce the volume fraction occupied by nucleus inside the WS cell, $w=4 \pi r_{p}^{3} /\left(3 V_{c}\right)$; the surface area of a nucleus, $\mathcal{A}=4 \pi r_{p}^{2}$; and the electron number density $n_{e}$, determined from the quasi-neutrality condition, $n_{e}=w n_{p i}$.
Within CLDM the energy density can be written as

$$
\begin{aligned}
\epsilon= & w \epsilon^{\text {bulk }}\left(n_{n i}, n_{p i}\right)+(1-w) \epsilon^{\text {bulk }}\left(n_{n o}, 0\right) \\
& +E_{s}\left(\nu_{s}, r_{p}\right) / V_{c}+E_{C}\left(n_{p i}, r_{p}, w\right) / V_{c}+\epsilon_{e}\left(n_{e}\right) .
\end{aligned}
$$

Here $\epsilon^{\text {bulk }}\left(n_{n}, n_{p}\right)$ is the energy density of homogeneous nuclear matter; $E_{s}$ is the surface energy of a nucleus. The Coulomb energy of a WS cell is given by $E_{C}=$ $\left(16 \pi^{2} / 15\right)\left(n_{p i} e\right)^{2} r_{p}^{5} f(w)$, where $f(w)=1-1.5 w^{1 / 3}+$ $0.5 \mathrm{w}$, and $\epsilon_{e}$ is the energy density of degenerate electron gas [1].

Taking the baryon number density, $n_{b}=w\left(n_{p i}+\right.$ $\left.n_{n i}\right)+(1-w) n_{n o}+\mathcal{A} \nu_{s} / V_{c}$, and number density of nuclei, $n_{N}=V_{c}^{-1}$, to be fixed, we minimize $\varepsilon$ with respect to other independent variables and obtain the beta-equilibrium, mechanical and local neutron diffusion equilibrium (within one unit cell) conditions. Using these conditions (see Supplementary material for more details), we arrive at the two-parameter equation of state, $\epsilon=\epsilon\left(n_{b}, n_{N}\right)$, with the second law of thermodynamics presented as

$$
\mathrm{d} \epsilon=\mu_{n} \mathrm{~d} n_{b}+\mu_{N} \mathrm{~d} n_{N},
$$

where $\partial \epsilon\left(n_{b}, n_{N}\right) / \partial n_{b}$ is denoted as $\mu_{n}$, because it equals the chemical potential of free (unbound) neutrons, as follows from the minimization procedure discussed above. The effective chemical potential $\mu_{N}$ describes the energy change due to addition of an extra nuclear cluster to the system at fixed $n_{b}, \mu_{N}=\left(\sigma \mathcal{A}-2 E_{C}\right) / 3$, where $\sigma$ is the surface tension [1] (see Supplementary material). Catalyzed EOS corresponds to absolute minimum of $\varepsilon$ at fixed $n_{b}$, hence it is given by the condition $\mu_{N}=0$. With this condition EOS becomes one-parametric, i.e., specified in a unique way for a given $n_{b}$.

For accreted crust $T$ is not high enough to allow for nuclear reactions that minimize $\varepsilon$ by choosing $n_{N}$ in an optimum way, thus $\mu_{N}$ is, generally, non-zero. To make EOS one-parametric we need an additional equation. In the traditional approach (e.g., Ref. [10]) the equation follows from the requirement that the total baryon number in the WS cell is conserved, $A_{c}=n_{b} V_{c}=$ const. (Note that this equation should be modified in the regions where pycnonuclear reactions proceed and $A_{c}$ doubles $[10,12-$ 14].)

And what about nHD EOS? $A_{c}$ is not conserved now, because neutrons can move independently of nuclei. Instead, this EOS should respect the nHD condition $\mu_{n}^{\infty}=$ const, as well as the general hydrostatic equilibrium condition, $P^{\prime}(r)=-(P+\epsilon) \nu^{\prime}(r) / 2[1]$, where $P$ is the pressure and prime $(')$ means derivative with respect to the radial coordinate $r$. Combining these two equations with the Gibbs-Duhem relation, $d P=n_{b} d \mu_{n}+n_{N} d \mu_{N}$, one arrives at the requirement $\mu_{\mathrm{N}}^{\infty}=\mu_{\mathrm{N}} e^{\nu / 2}=$ const. In other words (because $\mu_{n} e^{\nu / 2}$ is also a constant), the ratio $\mu_{\mathrm{N}} / \mu_{n}$ must be fixed in the inner crust, i.e., $\mu_{\mathrm{N}} / \mu_{n}=C$, or, recalling the definition of $\mu_{N}$,

$$
\sigma \mathcal{A}-2 E_{C}=3 C \mu_{n},
$$


where $C$ is some constant. This condition parametrizes a family of nHD EOSs. It allows, in particular, to present $\mu_{n}$ as a function of $P$ and $C: \mu_{n}=\mu_{n}(P, C)$. Catalyzed EOS is a member of this family (hence neutrons are in the diffusion equilibrium in catalyzed matter - an expected result); it corresponds to the choice $C=0$ (i.e., $\mu_{\mathrm{N}}=0$ ). As shown below, only one particular $C$ corresponds to the fully accreted NS crust, which we shall be mostly interested in what follows.

nHD EOS for a fully accreted crust. - In this case $C$ can be determined from two requirements: (i) $P$ and $\mu_{n}$ at the crust-core boundary must be continuous; and (ii) the structure and composition of fully accreted crust should not change in the course of accretion. In particular, the latter condition means that the total number of nuclei in the crust should be conserved. However, accretion permanently brings nuclei to the crust. Clearly, the stationary situation is possible only if the same amount of nuclei disintegrate somewhere in the crust.

nHD EOS provides a natural mechanism of nuclei disintegration due to a specific instability discussed below. Namely, numerical calculations show that, for each $C$ there is a maximum pressure $P_{\max }$, such that the solution to Eq. (3) does not exist at $P>P_{\max }$ (in Supplementary material we argue that it is a general feature of nHD EOSs).

To demonstrate the physical mechanism behind the instability we, first of all, combine the equation $P^{\prime}(r)=$ $-(P+\epsilon) \nu^{\prime}(r) / 2$ and condition $\mu_{n}^{\infty}=$ const to derive a relation, $d \mu_{n}=\mu_{n} /(P+\epsilon) d P$, which is valid in the nHDequilibrated inner crust and is equivalent to Eq. (3). It states that $\mu_{n}$ in a given volume is fixed if $P$ is fixed, independently of nuclear transformations occurring in this volume. Now, let us consider a layer, initially located at $P_{\max }$, but compressed slightly by newly accreted material, so that $P$ is a bit larger than $P_{\max }$. Absence of stationary solutions at $P>P_{\max }$ means that the layer should be out of beta-equilibrium at such pressure (otherwise it is impossible to remain in the hydrostatic equilibrium). Then beta-captures come into play trying to return the system to the beta-equilibrium, but, as we checked numerically, they are accompanied by neutron emissions and the emitted neutrons diffuse out of the layer in order to preserve $\mu_{n}$ at a given $P$. As a result, the layer begins to shrink and nuclei in the layer start to 'evaporate' ( $A$ and $Z$ decrease) until disintegration - the required instability.

This instability is, in fact, similar to the mechanism discussed in Ref. [28]. Namely, at $P>P_{\max }$ nuclei become unstable with respect to electron capture accompanied by emission of neutrons. Each electron capture makes nucleus even more unstable, leading to a series of subsequent electron captures and neutron emissions until complete disintegration. The instability is also analogous to the superthreshold electron capture cascades studied in Refs. [16, 17, 29], but, in contrast to these works, disin-

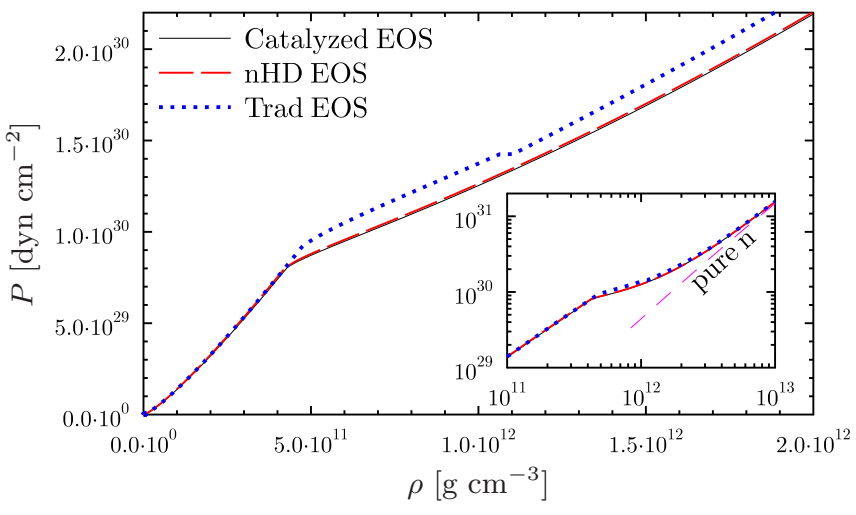

FIG. 1. Pressure versus density for different crustal EOSs discussed in the text.

tegration is complete and takes place at fixed $P$ and $\mu_{n}$.

During accretion, the number of nuclei in the (initially catalyzed) crust is increasing until the instability sets in at $P=P_{\max }$. Since at $P>P_{\max }$ stable crust does not exist, $P_{\max }$ should coincide with the pressure at the crust-core boundary [30]. Thus, the parameter $C$ and hence nHD EOS for a fully accreted crust (hereafter, simply 'nHD EOS') can be determined by matching $\mu_{n}$ at $P=P_{\max }$ in the crust and in the core: $\mu_{n}\left(P_{\max }, C\right)=\mu_{n}^{\text {core }}\left(P_{\max }\right)$, where $\mu_{n}^{\text {core }}(P)$ stands for $\mu_{n}$ in the core.

Now we have everything at hand to find where the outer-inner crust interface is located. To this end, we note that, by definition, one has $m_{n}=\mu_{n}$ at the interface, thus the pressure $P_{\text {oi }}$ there can be found from the condition: $m_{n}=\mu_{n}\left(P_{\mathrm{oi}}, C\right)$. Note that it should not necessarily coincide (for nHD EOS) with the neutron drip pressure $P_{\text {nd }}$ of Trad EOS, because the latter is obtained neglecting possible redistribution of neutrons in the star.

Numerical example. - To illustrate our results we employ SLY4 energy density functional [31]; the corresponding surface energy and tension $\sigma$ are adopted from Ref. [32]. We find that the interface between the inner and outer crust is located at $8.0 \times 10^{29}, 8.1 \times 10^{29}$, and

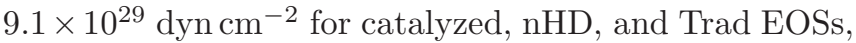
respectively (for $\mathrm{nHD}$ EOS such $P_{\text {oi }}$ leads to $C \approx 0.0025$ ). The corresponding pressures at the crust-core boundary equal $4.93 \times 10^{32}, 5.20 \times 10^{32}$, and $5.14 \times 10^{32} \mathrm{dyn} \mathrm{cm}^{-2}$. For simplicity, when considering Trad model, we assume that the pycnonuclear reactions take place at $Z=10$.

Fig. 1 demonstrates three EOSs described in this work: catalyzed (solid line), nHD (long dashes), and Trad (dots); EOS of pure neutron matter (dashed line) is added for comparison. One can see that nHD EOS, suggested here, significantly differs from Trad EOS, obtained within the traditional approach, and is much closer to the catalyzed EOS (cf., e.g., $P_{\text {oi }}$ for catalyzed and nHD EOSs: $8.0 \times 10^{29}$ and $8.1 \times 10^{29} \mathrm{dyn}_{\mathrm{cm}^{-2}}$ ).

The flat region at $\rho \sim 1.1 \times 10^{12} \mathrm{~g} \mathrm{~cm}^{-3}$ for Trad EOS 


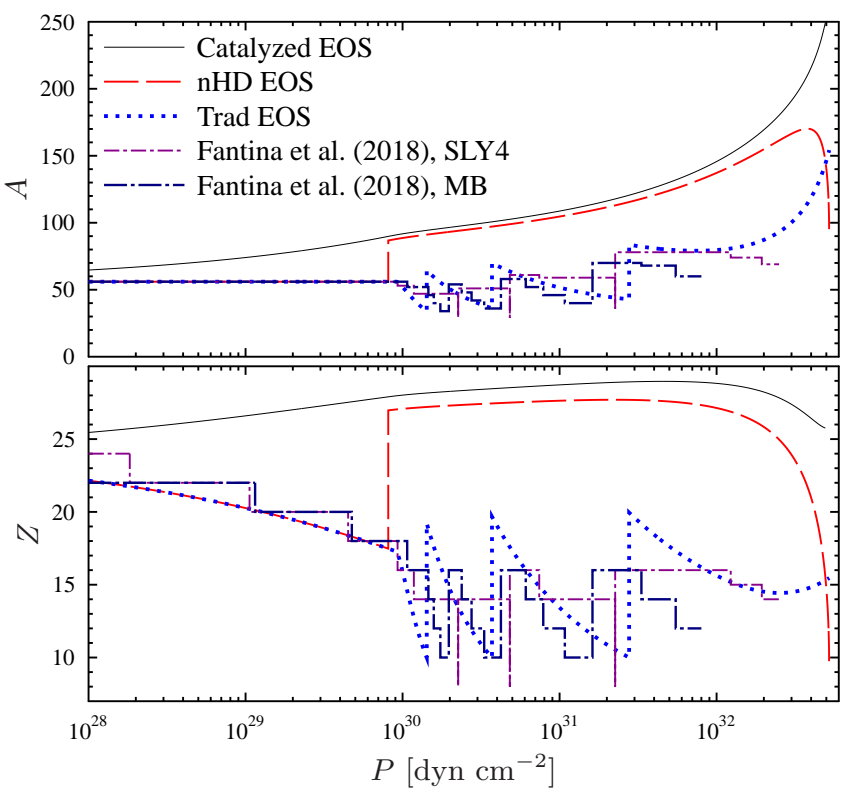

FIG. 2. The nuclei charge $Z$ and atomic mass number $A$ as function of pressure for different crustal EOSs.

corresponds to pycnonuclear reaction. These reactions are also clearly visible as jumps in Fig. 5, which demonstrates profiles of nuclear charges $Z$ and mass numbers $A$ for the same EOSs as in Fig. 1 [in Supplementary material we also show the function $\left.A_{c}(P)\right]$. In addition, dotdashed lines show profiles obtained in Ref. [14] ignoring the condition $\mu_{n}^{\infty}=$ const. The corresponding EOSs are calculated for Sly4 functional in the extended ThomasFermi approach and for liquid-drop model of Ref. [33]. One can see that for traditional approach our CLDM reproduces the results of Ref. [14] reasonably well, in particular, pycnonuclear reactions occur three times in the inner crust.

Crust composition (i.e., $Z$ and $A$ ) for nHD EOS is determined by Eq. (3). One may note that it is remarkably different from that for Trad EOS, being rather close (at not too large $P$ ) to the composition of catalyzed crust. The latter fact is not surprising, since at not too large $P$ two terms in the l.h.s. of Eq. (3) are much larger than the term $C \mu_{n}$ in its r.h.s., hence Eq. (3) is quite similar to its 'catalyzed' counterpart, $\mu_{N}=0$. At larger $P$ surface tension decreases, because matter inside and outside nuclear clusters becomes more and more similar, while the term $C \mu_{n}$ increases and, eventually, all three terms in Eq. (3) become comparable; as a result $Z$ and $A$ for nHD EOS substantially differ from those for catalyzed EOS at such $P$.

Discussion and conclusions. - We construct the model of the inner crust of accreting NS, which respects the nHD condition, $\mu_{n}^{\infty}=$ const, imposed by the requirement of hydrostatic equilibrium with respect to superfluid equation in the most part of the inner crust and by the diffusion equilibrium in a thin layer near the outerinner crust interface. We find that the resulting nHD EOS is rather close to the catalyzed one, being significantly different from Trad EOS obtained in the traditional approach, which ignores the condition $\mu_{n}^{\infty}=$ const and implicitly assumes that both nuclei and unbound neutrons move together with one and the same velocity. Our another important result is that we found an instability that allows to transform nuclei into npe-matter at the crust-core boundary, and explain its physical meaning. We also demonstrate that the interface $P=P_{\mathrm{oi}}$ between the (accreted) outer and inner crust is not associated with the 'standard' neutron drip pressure $P_{\text {nd }}$, at which neutrons 'drip out' of nuclei [34]. Instead, $P_{\text {oi }}$ is determined by the nHD equilibrium condition inside the star. As a result, $P_{\mathrm{oi}}$ in the accreted crust appears to be just a bit higher than in the catalyzed crust, and nuclei at the $P=P_{\text {oi }}$ interface absorb neutrons rather than emit them, as in Trad EOS. Neutron absorptions (accompanied by electron emissions) lead to a jump of $A$ at the upper boundary of the inner crust (see Fig. 5). Neutrons, necessary for such absorptions, are supplied by upward neutron flow, which originates at the crust-core boundary, where nuclei disintegrate into neutrons as a result of the instability discussed above. Then these neutrons redistribute over the inner crust and core in order to maintain $\mathrm{nHD}$ equilibrium.

Similarity of nHD and catalyzed EOSs suggests that accretion should have a less pronounced effect on the crust thickness and tidal deformability than in the traditional approach. It also suggests that the heat release due to nonequilibrium nuclear reactions in the accreted crust should be much smaller than it is usually thought to be, and this idea agrees with apparently very different reaction flows for nHD EOS (e.g., pycnonuclear reactions for nHD EOS are absent). The heat release problem is considered in detail in our forthcoming publication [35]. According to preliminary estimates, the net heat release is $\sim 0.5 \div 0.7 \mathrm{MeV} /$ nucleon (i.e., $2-3$ times smaller than in the traditional approach), with significant fractions released at the outer-inner crust interface and crust-core boundary. These findings, along with modification of the transport properties and heat capacity (due to changed nuclear composition), should noticeably affect interpretation of transiently accreting NSs and may shed new light on the shallow heating and superburst ignition problems (e.g., [7]). The detailed analysis is left for future work.

The crucial role of the neutron hydrostatic and diffusion equilibrium for accreted crust EOS, revealed in this Letter, is a general feature, which can not be disregarded (see also [20]). However, we should warn the reader that our results are illustrated within the simplified CLDM, which treats nuclear mass and charge numbers as continuous variables and neglects pairing and shell effects. In Ref. [14] the latter are shown to be important for the energy release in the traditional approach. According to our 
preliminary results, obtained within the nHD approach, shell effects mainly influence profile of the heat release and composition of the crust; at the same time, the total heat release and $P(\rho)$ dependence are not strongly affected.

We are grateful to E.M. Kantor and N.N. Shchechilin for numerous useful discussions. Work is supported by Russian Science Foundation (grant 19-12-00133).

[1] P. Haensel, A. Potekhin, and D. Yakovlev, Neutron Stars 1: Equation of State and Structure, Astrophysics and Space Science Library (Springer-Verlag, Berlin, 2006).

[2] N. Chamel and P. Haensel, Liv. Rev. Relativ. 11, 10 (2008).

[3] C. O. Heinke, P. G. Jonker, R. Wijnands, C. J. Deloye, and R. E. Taam, Astrophys. J. 691, 1035 (2009), arXiv:0810.0497.

[4] R. Wijnands, N. Degenaar, and D. Page, Journal of Astrophysics and Astronomy 38, 49 (2017).

[5] Y. Zhao, C. O. Heinke, S. S. Tsygankov, W. C. G. Ho, A. Y. Potekhin, and A. W. Shaw, Mon. Not. R. Astron. Soc. 488, 4427 (2019), arXiv:1907.04969 [astro-ph.HE].

[6] A. Y. Potekhin, A. I. Chugunov, and G. Chabrier, arXiv eprints, arXiv:1907.08299 (2019), arXiv:1907.08299 [astroph.HE].

[7] Z. Meisel, A. Deibel, L. Keek, P. Shternin, and J. Elfritz, ArXiv e-prints (2018), arXiv:1807.01150 [astro-ph.HE].

[8] M. E. Gusakov, E. M. Kantor, and A. Reisenegger, Mon. Not. R. Astron. Soc. 453, L36 (2015), arXiv:1507.04586 [astro-ph.HE].

[9] K. Sato, Progress of Theoretical Physics 62, 957 (1979).

[10] P. Haensel and J. L. Zdunik, Astron. Astrophys. 227, 431 (1990).

[11] P. Haensel and J. L. Zdunik, Astron. Astrophys. 229, 117 (1990).

[12] P. Haensel and J. L. Zdunik, Astron. Astrophys. 404, L33 (2003), astro-ph/0305220.

[13] P. Haensel and J. L. Zdunik, Astron. Astrophys. 480, 459 (2008), arXiv:0708.3996.

[14] A. F. Fantina, J. L. Zdunik, N. Chamel, J. M. Pearson, P. Haensel, and S. Goriely, Astron. Astrophys. 620, A105 (2018), arXiv:1806.03861 [astro-ph.HE].

[15] A. W. Steiner, Phys. Rev. C 85, 055804 (2012), arXiv:1202.3378 [nucl-th].

[16] R. Lau, M. Beard, S. S. Gupta, H. Schatz, A. V. Afanasjev, E. F. Brown, A. Deibel, L. R. Gasques, G. W. Hitt, W. R. Hix, L. Keek, P. Möller, P. S. Shternin, A. W. Steiner, M. Wiescher, and Y. Xu, Astrophys. J. 859, 62 (2018), arXiv:1803.03818 [astro-ph.HE].

[17] N. N. Shchechilin and A. I. Chugunov, Monthly Notices of the Royal Astronomical Society 490, 3454 (2019), http://oup.prod.sis.lan/mnras/articlepdf/490/3/3454/30338149/stz2838.pdf.

[18] N. N. Shchechilin and A. I. Chugunov, Journal of Physics: Conference Series, 1400, 022016 (2019), arXiv:2001.09739 [astro-ph.SR].

[19] The only exception, which allows for diffusion of unbound neutrons is a series of works $[24,36]$, but it mainly focuses on the crust properties of newly born NSs (see also Discussion section in Ref. [15]).

[20] A. I. Chugunov and N. N. Shchechilin, MNRAS Lett., accepted (2020).

[21] I. M. Khalatnikov, An Introduction to the Theory of Superfluidity (Addison-Wesley, New York, 1989).

[22] M. E. Gusakov and N. Andersson, Mon. Not. R. Astron. Soc. 372, 1776 (2006).

[23] C. J. Pethick, N. Chamel, and S. Reddy, Progress of Theoretical Physics Supplement 186, 9 (2010), arXiv:1009.2303 [astro-ph.SR].

[24] G. S. Bisnovatyi-Kogan and V. M. Chechetkin, Uspekhi Fizicheskikh Nauk 127, 263 (1979).

[25] J. M. Lattimer, C. J. Pethick, D. G. Ravenhall, and D. Q. Lamb, Nucl. Phys. A 432, 646 (1985).

[26] F. Douchin and P. Haensel, Physics Letters B 485, 107 (2000), arXiv:astro-ph/0006135 [astro-ph].

[27] X. Viñas, C. Gonzalez-Boquera, B. K. Sharma, and M. Centelles, Acta Physica Polonica B, Proceedings Supplement 10, 259 (2017).

[28] G. S. Bisnovatyi-Kogan and V. M. Chechetkin, Astrophys. Space Sci. 26, 25 (1974).

[29] S. Gupta, E. F. Brown, H. Schatz, P. Möller, and K.-L. Kratz, Astrophys. J. 662, 1188 (2007), astro-ph/0609828.

[30] This is strictly true for CLDM employed here. For a more realistic models with integer $A$ and $Z$ and, possibly, with nonspherical nuclei [37] near the crust-core boundary, it may happen that the instability occurs earlier, e.g., at the phase transition between the (standard) spherical nuclei and cylindrical nuclear shapes.

[31] E. Chabanat, P. Bonche, P. Haensel, J. Meyer, and R. Schaeffer, Nucl. Phys. A 635, 231 (1998).

[32] F. Douchin, P. Haensel, and J. Meyer, Nucl. Phys. A 665, 419 (2000).

[33] F. D. Mackie and G. Baym, Nucl. Phys. A 285, 332 (1977).

[34] N. Chamel, A. F. Fantina, J. L. Zdunik, and P. Haensel, Phys. Rev. C 91, 055803 (2015), arXiv:1504.04537 [astroph.HE].

[35] M. E. Gusakov and A. I. Chugunov, to be submitted.

[36] G. S. Bisnovatyi-Kogan, Y. N. Kulikov, and V. M. Chechetkin, Sov. Astron. 20, 552 (1976).

[37] D. G. Ravenhall, C. J. Pethick, and J. R. Wilson, Phys. Rev. Lett. 50, 2066 (1983). 


\section{Supplementary material}

\section{Compressible liquid-drop model}

In the Supplementary material we present more details about compressible liquid drop model (CLDM), used in the Letter. For the reader's convenience, the text of the supplementary material is made self-contained.

\section{Energy density}

Within the CLDM nuclei (clusters of nucleons) are described as a spherically symmetric liquid drops of nuclear matter, located at the center of spherical electrically neutral Wigner-Seitz (WS) cell [1-3]. The model is parametrized by six parameters: $n_{n i}, n_{p i}$, and $n_{n o}$ are the number densities of neutrons and protons inside, and neutrons outside nucleus, respectively (for simplicity, we assume that proton drip does not occur in the crust, which is true for all numerical models discussed in the Letter); $\nu_{s}$ is the neutron surface density in the neutron skin; $V_{c}, r_{p}$ are the WS cell volume and (proton) radius of a nucleus, respectively (with such definition of the nucleus radius the proton skin vanishes, see, e.g., appendix B in Ref. [1] and Ref. [4]).

In addition, it is useful to introduce the volume fraction occupied by nucleus inside the WS cell,

$$
w=\frac{4 \pi r_{p}^{3}}{3 V_{c}}
$$

the surface area of a nucleus, $\mathcal{A}=4 \pi r_{p}^{2}$; and the electron number density, $n_{e}$, which is determined by the quasineutrality condition, $n_{e}=w n_{p i}$. In what follows, we make use of the continuous CLDM, i.e., we treat the charge $Z=n_{e} V_{c}$ and mass number $A=Z+4 \pi n_{n i} r_{p}^{3} / 3+\mathcal{A} \nu_{s}$ of nuclei as continuous variables, neglecting pairing and shell effects. The energy density for CLDM is written as (see, e.g., Ref. [2])

$$
\epsilon=w \epsilon^{\mathrm{bulk}}\left(n_{n i}, n_{p i}\right)+(1-w) \epsilon^{\mathrm{bulk}}\left(n_{n o}, 0\right)+\frac{E_{s}\left(\nu_{s}, r_{p}\right)}{V_{c}}+\frac{E_{C}\left(n_{p i}, r_{p}, w\right)}{V_{c}}+\epsilon_{e}\left(n_{e}\right) .
$$

Here $\epsilon^{\text {bulk }}\left(n_{n}, n_{p}\right)$ is the energy density as a function of neutron and proton number densities. For numerical estimates we adopt SLY4 energy-density functional [5]; in the Letter the same functional is also used to calculate equation of state (EOS) in the neutron star (NS) core. In Eq. (5) $E_{C}$ is the Coulomb energy,

$$
E_{C}=\frac{16 \pi^{2}}{15}\left(n_{p i} e\right)^{2} r_{p}^{5} f(w)=\frac{3}{5} \frac{Z^{2} e^{2}}{r_{p}} f(w),
$$

where $f(w)=1-1.5 w^{1 / 3}+0.5 w ; e$ and $\epsilon_{e}$ is the electric charge and energy density of degenerate electron gas, respectively [2]. The electron gas is assumed to be ideal in all numerical calculations.

The surface energy is described, in a thermodynamically-consistent way, as (see, e.g., Refs. $[1,2,6]$ )

$$
E_{s}=\mathcal{A}\left(\sigma+\mu_{n s} \nu_{s}\right)
$$

where $\sigma$ and $\mu_{n s}$ are the so called surface tension and chemical potential for neutrons absorbed at the nucleus surface. For simplicity, when calculating these quantities, we neglect curvature corrections, related to the fact that nucleus surface is a sphere rather than a plain. Then $\sigma$ and $\mu_{n s}$ are the only functions of $\nu_{s}: \sigma=\sigma\left(\nu_{s}\right), \mu_{n s}=\mu_{n s}\left(\nu_{s}\right)[1,2]$. Introducing the total number of neutrons absorbed at the nucleus surface, $N_{s}=\mathcal{A} \nu_{s}$, the neutron chemical potential $\mu_{n s}$ can be presented as

$$
\mu_{n s}=\left.\frac{\partial E_{s}}{\partial N_{s}}\right|_{\mathcal{A}}=\frac{d}{d \nu_{s}}\left(\sigma+\mu_{n s} \nu_{s}\right) .
$$

This expression implies a relation of thermodynamical consistency for the function $\sigma\left(\nu_{s}\right)$

$$
\frac{d \sigma}{d \nu_{s}}=-\nu_{s} \frac{d \mu_{n s}}{d \nu_{s}}
$$

For numerical estimates we extract $\sigma\left(\nu_{s}\right)$ and $\mu_{n s}\left(\nu_{s}\right)$ from the results of Ref. [4]. Because both $\sigma$ and $\mu_{n s}$ are functions of $\nu_{s}$, one can also present $\sigma$ as a function of $\mu_{n s}: \sigma=\sigma\left(\mu_{n s}\right)$ (see Fig. 3), which is especially suitable for numerical applications. 


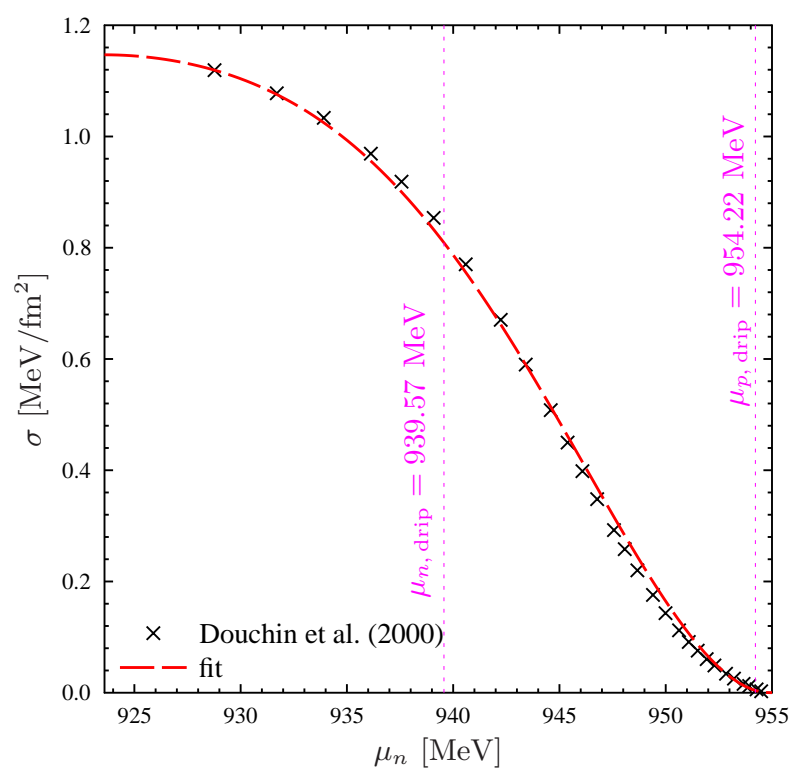

FIG. 3. Surface tension $\sigma$ versus $\mu_{n s}$. Dashed line corresponds to the fit used in the Letter; crosses show the data extracted from Ref. [4]. Thin vertical lines indicate neutron and proton drips.

\section{Differential of the energy density and reduction to the two parameter EOS}

To write down a differential of the energy density, $d \varepsilon$, in a compact form [7], let us present $\epsilon$ as function of $n_{n i}^{\text {(tot) }}$, $n_{p i}^{\text {(tot) }}, n_{n o}^{\text {(tot) }}, n_{s}^{(\text {tot })}, n_{N}$, and $w: \epsilon=\epsilon\left(n_{n i}^{(\text {tot })}, n_{p i}^{(\text {tot })}, n_{n o}^{(\text {tot })}, n_{n s}^{(\text {tot })}, n_{N}, w\right)$, where the auxiliary variables are:

$$
\begin{aligned}
n_{n i}^{(\mathrm{tot})} & \equiv n_{n i} w, \\
n_{p i}^{(\mathrm{tot})} & \equiv n_{p i} w, \\
n_{n o}^{(\mathrm{tot})} & \equiv n_{n o}(1-w), \\
n_{n s}^{(\mathrm{tot})} & \equiv \frac{N_{s}}{V_{\text {cell }}} \\
n_{N} & =V_{c}^{-1} .
\end{aligned}
$$

They have a simple physical meaning. For example, $n_{n i}^{(\text {tot })}$ is the number of neutrons in a nucleus per WS cell volume $V_{c}$, and $n_{\mathrm{N}}$ is the number density of nuclei. Using these variables, the baryon number density reads

$$
n_{b}=n_{n i}^{(\mathrm{tot})}+n_{p i}^{(\mathrm{tot})}+n_{n o}^{(\mathrm{tot})}+n_{n s}^{(\mathrm{tot})}
$$

while the quasineutrality condition is simply $n_{e}=n_{p i}^{(\text {tot })}$. In terms of these variables $d \varepsilon$ can be written as

$$
d \epsilon=\frac{\partial \epsilon}{\partial n_{n i}^{(\mathrm{tot})}} d n_{n i}^{(\mathrm{tot})}+\frac{\partial \epsilon}{\partial n_{p i}^{(\mathrm{tot})}} d n_{p i}^{(\mathrm{tot})}+\frac{\partial \epsilon}{\partial n_{n o}^{(\mathrm{tot})}} d n_{n o}^{(\mathrm{tot})}+\frac{\partial \epsilon}{\partial n_{n s}^{(\mathrm{tot})}} d n_{n s}^{(\mathrm{tot})}+\frac{\partial \epsilon}{\partial n_{N}} d n_{N}+\frac{\partial \epsilon}{\partial w} d w
$$

or, after straightforward calculation of derivatives using Eq. (5), as

$$
\begin{aligned}
d \epsilon & =\mu_{n i} d n_{n i}^{(\mathrm{tot})}+\left(\mu_{p i}+\mu_{e}+\frac{2}{Z} E_{C}\right) d n_{p i}^{(\mathrm{tot})}+\mu_{n o} d n_{n o}^{(\mathrm{tot})}+\mu_{n s} d n_{n s}^{(\mathrm{tot})} \\
& +\frac{1}{3}\left(\sigma \mathcal{A}-2 E_{\mathrm{Coul}}\right) d n_{N}+\left[P_{o}^{\mathrm{bulk}}-P_{i}^{\mathrm{bulk}}+\frac{2 \sigma}{r_{p}}-\frac{(1-w) n_{N}}{3 w f(w)} E_{C}\right] d w
\end{aligned}
$$


where we defined

$$
\begin{aligned}
\mu_{n i} & =\mu_{n i}^{\text {bulk }} \equiv \frac{\partial \epsilon^{\mathrm{bulk}}\left(n_{n i}, n_{p i}\right)}{\partial n_{n i}}, \quad \mu_{n o}=\mu_{n o}^{\text {bulk }} \equiv \frac{\partial \epsilon^{\mathrm{bulk}}\left(n_{n o}, 0\right)}{\partial n_{n o}}, \\
\mu_{p i} & =\mu_{p i}^{\text {bulk }} \equiv \frac{\partial \epsilon^{\mathrm{bulk}}\left(n_{n i}, n_{p i}\right)}{\partial n_{p i}}, \\
\mu_{e} & =\frac{\partial \epsilon_{e}\left(n_{e}\right)}{\partial n_{e}} \\
P_{i}^{\text {bulk }} & =-\epsilon^{\text {bulk }}\left(n_{n i}, n_{p i}\right)+\mu_{p i}^{\text {bulk }} n_{p i}+\mu_{n i}^{\text {bulk }} n_{n i}, \\
P_{o}^{\text {bulk }} & =-\epsilon^{\text {bulk }}\left(n_{n o}, 0\right)+\mu_{n o}^{\text {bulk }} n_{n o} .
\end{aligned}
$$

Now, expressing $n_{n i}^{\text {(tot) }}$ through $n_{b}$ using Eq. (15), Eq. (17) can be rewritten as

$$
\begin{aligned}
d \epsilon & =\mu_{n i} d n_{b}+\left(\frac{2 E_{C}}{Z}+\mu_{p i}+\mu_{e}-\mu_{n i}\right) d n_{p i}^{(\text {tot })}+\left(\mu_{n o}-\mu_{n i}\right) d n_{n o}^{(\text {tot })}+\left(\mu_{n s}-\mu_{n i}\right) d n_{n s}^{(\text {tot })} \\
& +\frac{1}{3}\left(\sigma \mathcal{A}-2 E_{\text {Coul }}\right) d n_{N}+\left[P_{o}^{\text {bulk }}-P_{i}^{\text {bulk }}+\frac{2 \sigma}{r_{p}}-\frac{(1-w) n_{N}}{3 w f(w)} E_{C}\right] d w .
\end{aligned}
$$

This equation is ideally suited to minimize the energy density at fixed $n_{b}$ and $n_{N}$ with respect to other variables, e.g., $n_{p i}^{\text {(tot) }}, n_{n o}^{(\text {tot })}, n_{n s}^{\text {(tot) }}$, and $w$. It leads to two-parameter EOS, $\epsilon\left(n_{b}, n_{N}\right)$, discussed in the Letter. Other variables of CLDM, required to calculate $\varepsilon$ via Eq. (5), can be determined, for given $n_{b}$ and $n_{N}$, from the following equations [see Eq. (23)]:

1) Beta-equilibrium condition (minimization of $\epsilon$ with respect to $n_{p i}^{(\text {tot })}$ ):

$$
\mu_{n i}=\mu_{p i}+\mu_{e}+\frac{2 E_{C}}{Z}
$$

2) Neutron local diffusion equilibrium inside the WS cell (minimization of $\epsilon$ with respect to $n_{n o}^{(\text {tot })}$ and $n_{n s}^{(\text {tot })}$ )

$$
\mu_{n i}=\mu_{n o}=\mu_{n s}
$$

3) The pressure balance equation (minimization of $\epsilon$ with respect to $w$ )

$$
P_{i}^{\text {bulk }}=P_{o}^{\text {bulk }}+\frac{2 \sigma}{r_{p}}-\frac{(1-w) n_{N}}{3 w f(w)} E_{C} .
$$

According to Eq. (25), the neutron chemical potential is constant in all parts of a WS cell (inside and outside of a nucleus, as well as in the neutron skin), as it should be in thermodynamic equilibrium. Thus, when considering the two-parameter EOS, $\epsilon\left(n_{b}, n_{N}\right)$, it is reasonable to suppress unnecessary indices and denote the neutron chemical potential simply as $\mu_{n}$. Using Eqs. (24)-(26), the expression (23) reduces to

$$
d \epsilon=\mu_{n} d n_{b}+\mu_{N} d n_{N}
$$

where we introduced a new parameter,

$$
\mu_{N}=\frac{1}{3}\left(\sigma \mathcal{A}-2 E_{C}\right)
$$

which can be interpreted as an effective chemical potential for nuclear clusters. It describes how the system energy will change if we create there an additional nuclear cluster, keeping, at the same time, the total baryon number in the system fixed.

\section{Pressure}

To finalize construction of EOS we should determine the pressure $P$. According to the second law of thermodynamics, it is equal to (minus) derivative of the system energy with respect to volume $V$ at fixed total particle numbers 
$n_{n b} V, n_{p i}^{(\text {tot })} V, n_{n o}^{(\text {tot })} V, n_{n s}^{(\text {tot })} V$, and $n_{N} V$. Thus, in the most general case, using Eq. (23), the pressure can be written as

$$
P \equiv-\frac{\partial(\epsilon V)}{\partial V}=-\epsilon+\mu_{n i} n_{b}+\left(\frac{2 E_{C}}{Z}+\mu_{p i}+\mu_{e}-\mu_{n i}\right) n_{p i}^{(\mathrm{tot})}+\left(\mu_{n o}-\mu_{n i}\right) n_{n o}^{(\mathrm{tot})}+\left(\mu_{n s}-\mu_{n i}\right) n_{n s}^{(\mathrm{tot})}+\mu_{N} n_{N} .
$$

For a two-parameter EOS, $\epsilon\left(n_{b}, n_{N}\right)$, determined by the minimization procedure discussed above, $P$ reduces to [see Eqs. (24)-(26)]

$$
P=-\epsilon+\mu_{n} n_{b}+\mu_{N} n_{N}
$$

\section{Absence of solutions at $P>P_{\max }$ as a generic feature of equation (3) from the Letter}

Let us start with equation (3) from the Letter that should be used to determine $n_{\mathrm{N}}$ for a given $n_{b}$ for neutron Hydrostatic and Diffusion (nHD) EOS,

$$
\sigma \mathcal{A}-2 E_{C}=3 C \mu_{n}
$$

In fact, in what follows it will be more convenient to solve it for the nucleus radius $r_{p}$ (rather than $n_{N}$ ), and then find $n_{N}$ from the formula (4) and the relation $n_{N}=1 / V_{c}$. Using the expression (6) for $E_{C}$, Eq. (31) can be rewritten as

$$
\sigma\left(\mu_{n}\right)=\frac{8 \pi}{15} n_{p i}^{2} e^{2} f(w) r_{p}^{3}+\frac{3 C \mu_{n}}{4 \pi r_{p}^{2}}
$$

where the surface tension $\sigma$ is presented as the (known) function of the neutron chemical potential, $\mu_{n}=\mu_{n s}$ (see Fig. 3 and a comment at the end of Sec. ).

The quantities $w, n_{p i}$, and $\mu_{n}$ in Eq. (32) can be thought of as already expressed, using Eqs. (24)-(26), as some functions of $r_{p}$ and $n_{b}$. Thus, Eq. (32) is, generally, an implicit equation for $r_{p}$, which is difficult to solve and analyze. However, the analysis can be substantially simplified if one notes that such quantities as $n_{n i}, n_{p i}, n_{n o}, w, \mu_{n}$ can be found, to a good precision, from the so called bulk approximation, which ignores surface and Coulomb effects (see, e.g., Ref. [4]). In other words, these quantities should be approximately the same, e.g., for catalyzed matter and for nHD EOS we are interested in (these EOSs do not differ on the level of bulk approximation). Therefore, for a qualitative analysis of Eq. (32) we can present $w$ and $n_{p i}$ in Eq. (32) as the same functions of $\mu_{n}$ as for catalyzed matter. Since in this approximation $w$ and $n_{p i}$ do not depend on $r_{p}$, Eq. (32) can now be readily solved for $r_{p}$. However, we do not really need this solution in order to demonstrate that it does not have roots at some $P>P_{\max }$, or, equivalently, at $\mu_{n}>\mu_{n \max }$ (because $\mu_{n}$ is a growing function of $P$ for nHD EOS). To do that, note that the surface tension $\sigma\left(\mu_{n}\right)$ [the function in the left-hand side of Eq. (32)] is a decreasing function of $\mu_{n}$ (see Fig. 3), since nuclear matter inside and outside nuclear clusters becomes more and more similar with growing density [4]. At the same time, the right-hand side of Eq. (32) has a minimum as a function of $r_{p}$ at a point

$$
r_{p 0}=\left(\frac{15 C \mu_{n}}{16 \pi^{2} n_{p i}^{2} e^{2} f(w)}\right)^{1 / 5} .
$$

The corresponding minimum value of the right-hand side [let us denote it $F\left(\mu_{n}\right)$ ], which is a function of $\mu_{n}$, is given by

$$
F\left(\mu_{n}\right)=\left(\frac{125 C^{3}}{36 \pi} n_{p i}^{4} e^{4} f^{2}(w) \mu_{n}^{3}\right)^{1 / 5}
$$

and is a growing function of $\mu_{n}$. Correspondingly, because the left-hand side of Eq. (32) decreases, while the right-hand side increases with $\mu_{n}$, the maximum reachable value of the neutron chemical potential in the crust, $\mu_{n}=\mu_{n \max }$, is a solution to the equation

$$
\sigma\left(\mu_{n}\right)=F\left(\mu_{n}\right) .
$$

At $\mu_{n}>\mu_{n \max }$ the solution does not exist and we have an instability discussed in the Letter. Note that $n_{p i}$ and $w$ in this equation are (known) functions of $\mu_{n}$. Numerical solution to Eq. (35) is shown by dashes in Fig. 4, where we also present the function $\mu_{n \max }(C)$ obtained directly from equation (31) [or (32)] without any approximations (solid line). As one may see the agreement between the curves is rather good, which additionally justifies the approach described above. 


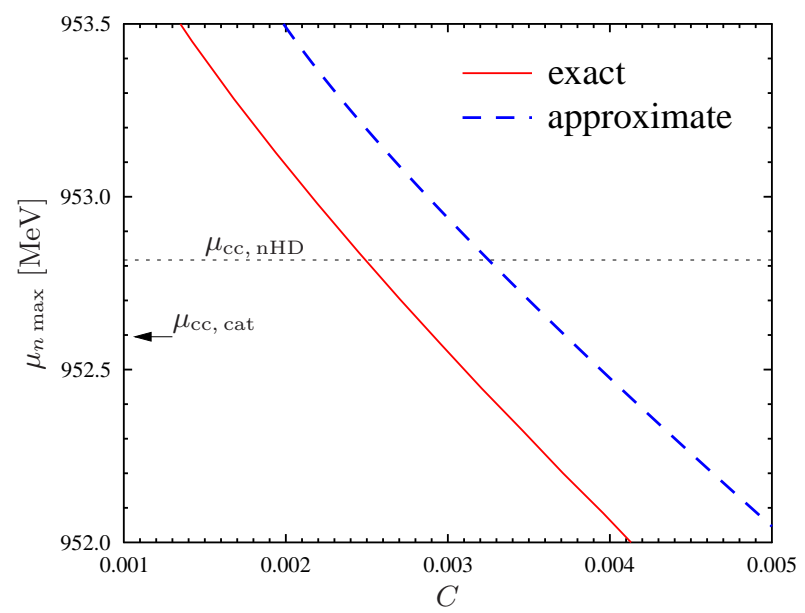

FIG. 4. Maximum reachable neutron chemical potential in the crust, $\mu_{n \max }$, as a function of the parameter $C$. Dashes: $\mu_{\mathrm{n} \max }(C)$ is obtained using the approximate method described in the text. Solid line: $\mu_{\mathrm{n} \max }(C)$ is numerically calculated from Eq. (31) without any approximations. Dotted horizontal line and arrow show $\mu_{n}$ at the crust-core boundary for the fully accreted $\mathrm{nHD} \operatorname{EOS}\left(\mu_{n}=\mu_{\mathrm{cc}, \mathrm{nHD}}\right)$ and for catalyzed crust $\left(\mu_{n}=\mu_{\mathrm{cc}, \mathrm{cat}}\right)$, respectively.

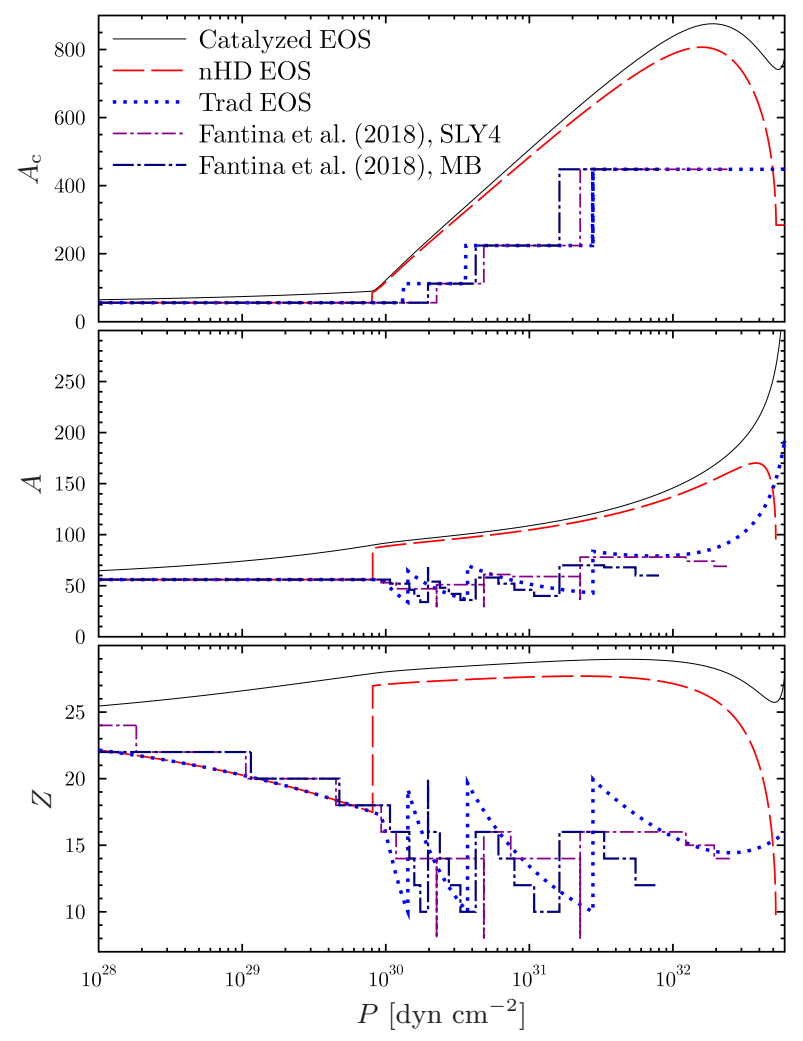

FIG. 5. The nuclei charge $Z$, atomic mass number $A$ and total number of nucleons in a WS cell, $A_{c}$, as function of pressure for different crustal EOSs discussed in the Letter.

\section{Crustal composition for different EOSs}

In Fig. 5 we present profiles of the crustal composition for several EOSs discussed in the Letter. The figure is analogue of figure 2 from the main text, with additional panel showing the total number of nucleons in a WS cell, $A_{c}$. 
[1] J. M. Lattimer, C. J. Pethick, D. G. Ravenhall, and D. Q. Lamb, Nucl. Phys. A 432, 646 (1985).

[2] P. Haensel, A. Potekhin, and D. Yakovlev, Neutron Stars 1: Equation of State and Structure, Astrophysics and Space Science Library (Springer-Verlag, Berlin, 2006).

[3] N. Chamel and P. Haensel, Liv. Rev. Relativ. 11, 10 (2008).

[4] F. Douchin, P. Haensel, and J. Meyer, Nucl. Phys. A 665, 419 (2000).

[5] E. Chabanat, P. Bonche, P. Haensel, J. Meyer, and R. Schaeffer, Nucl. Phys. A 635, 231 (1998).

[6] L. D. Landau and E. M. Lifshitz, Course of theoretical physics, Pergamon International Library of Science, Technology, Engineering and (Pergamon Press, Oxford, 1980).

[7] Using Eq. (5), it is straightforward to present $d \varepsilon$ in terms of original variables $\left(n_{n i}, n_{p i}, n_{n o}, w, V_{C}, \nu_{s}\right)$, but the resulting equations will be rather lengthly. 